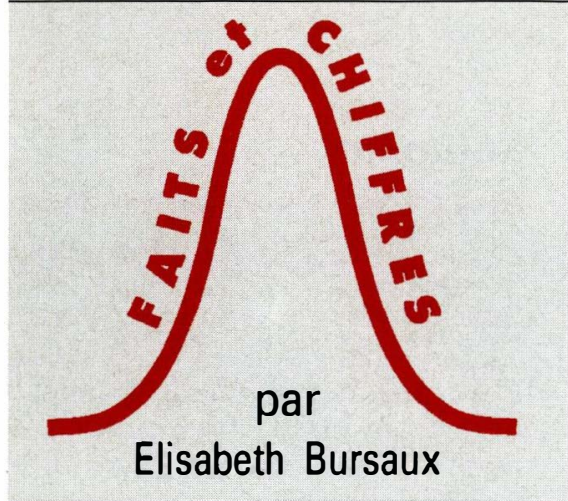

a plupart des mesures qui sont faites de l'importance relative des différentes maladies considèrent le nombre de décès qui leur sont imputables. Cette convention a certains avantages: le décès est un fait démographique bien défini, et de nombreux pays tiennent régulièrement des statistiques de mortalité. Cependant, de nombreuses maladies ou affections, sans être mortelles, réduisent considérablement la durée de vie valide de ceux qui en sont atteints : il en est ainsi, par exemple, de l'état dépressif chronique et de la paralysie causée par la poliomyélite. Ces affections sont répandues, elles peuvent durer longtemps et elles mettent souvent fortement à contribution les systèmes de santé. Afin de quantifier aussi précisément que possible l'impact de ces affections sur la durée de vie valide, la Banque mondiale et l'()'ganisation mondiale de la santé ont entrepris une étude conjointe qui a consisté à classer les maladies et iffections en 109 catégories d'après la Classification internationale des maladies (neuvième révision). Ces catégories comprennent toutes les causes possibles de décès et environ $95 \%$ des causes possibles d'invalidité. Sur la base des déclarations de décès lorsqu'elles en spécifiaient la cause ou, lorsque tel n'était pas le cas, d'estimations d'experts, tous les décès enregistrés en 1990 ont été répartis entre les 109 causes possibles et ventilés selon l'âge, le sexe et la région démographique. On a calculé la réduction de la durée de vie résultant de chaque décès en déduisant l'âge au décès de l'espérance de vie à cet âge dans une population à faible mortalité. Pour ce qui

\title{
Mesurer la charge de morbidité globale du Monde
}

est des cas d'invalidité, leur incidence selon l'âge, le sexe et la région démographique a été calculée d'après les résultats d'enquêtes auprès des populations ou, à défaut, sur la base d'évaluations d'experts; dans chaque cas, la réduction du nombre d'années de vie valide a été calculée en multipliant la durée probable de la maladie ou de l'affection (jusqu'à la rémission ou au décès) par un coefficient de pondération exprimant le degré de gravité de l'invalidité par rapport à la perte de la vie. Les maladies ont été rangées en six classes selon le degré d'invalidité qu'elles entraînent ; par exemple, on a assigné un coefficient de gravité de 0,22 aux affections de la classe 2, qui comprend la plupart des cas de lèpre et $50 \%$ des affections inflammatoires pelviennes, et un coefficient de gravité de 0,6 aux affections de la classe 4, qui comprend $30 \%$ des cas de démence et $50 \%$ des cas d'affections entraînant la cécité. On a ensuite ajouté dans chaque cas la réduction de la durée de la vie à la réduction pondérée de la durée de vie valide, en appliquant un taux d'actualisation de $3 \%$ (qui rend dégressive, avec le temps, la valeur assignée aux années futures de vie valide) et un coefficient de pondération selon l'âge (qui a pour effet de moduler en fonction de l'âge au décès la valeur attribuée aux années de vie perdues). La valeur de chaque année de vie perdue, indiquée dans la partie gauche de la figure 1, qui est nulle à la naissance, augmente ensuite rapidement jusqu'à atteindre un maximum pour l'âge de 25 ans, et diminue ensuite progressivement. Les coefficients de pondération selon l'âge reflètent le consensus d'un certain nombre d'experts, mais on aurait pu évidemment recourir à d'autres formules d'évaluation: par exemple, ne pas tenir compte de l'âge au décès et donc attribuer la même valeur à chacune des années de vie perdues, ce qui aurait accru l'importance relative des maladies de l'enfance. Ainsi, en appliquant un taux d'actualisation et un coefficient de pondération selon l'âge, on a calculé, pour les différents âges au décès, la réduction du nombre d'AVCI (années de vie corrigées du facteur invalidité). Comme il ressort de la partie droite de la figure 1, le décès d'un nouveau-né de sexe féminin représente une perte de 32,5 AVCI; le décès d'une femme de 30 ans entraîne une perte de 29 AVCI, et celui d'une femme de 60 ans une perte de 12 AVCI (les valeurs calculées pour les individus du sexe masculin sont légèrement inférieures). La réduction totale du nombre d'AVCI pour tous les âges, toutes les affections et toutes les régions représente la charge de morbidité globale. L'indicateur de la charge de morbidité globale mesure la valeur actualisée des années futures de vie valide perdues par suite des décès, des maladies et des affections traumatiques enregistrés en 1990. Ainsi, cet indicateur repose sur des faits survenus en 1990, mais rend compte de l'incidence future de ces faits sur le nombre d'années de vie valide. Le présent rapport exprime la charge de morbidité de trois manières différentes: par le nombre d'AVCI perdues, sous forme de pourcentage d'un agrégat quelconque (comme le pourcentage de perte totale imputable à telle maladie), et par rapport à l'effectif de la population en 1990. Ce dernier indicateur doit être interprété avec prudence, parce qu'il rapporte toutes les pertes futures à l'effectif actuel de la population et que la mesure peut fort bien dépasser une année par personne. Ainsi, le décès d'un nourrisson enregistré en 1990 apporte 32 ans à la charge de morbidité (valeur actualisée d'environ 80 ans d'espérance de vie), mais le nourrisson ne compte évidemment que pour une unité dans la popula- 
Tableau I

VENTILATION DES PERTES D'ANNÉES DE VIE CORRIGÉES DU FACTEUR INVALIDITÉ PAR CAUSE ET PAR RÉGION DÉMOGRAPHIQUE (1990)

\begin{tabular}{|c|c|c|c|c|c|c|c|c|c|}
\hline Cause & Monde & $\begin{array}{c}\text { Afrique } \\
\text { sub- } \\
\text { saharienne }\end{array}$ & Inde & Chine & $\begin{array}{l}\text { Autres } \\
\text { économies } \\
\text { et îles } \\
\text { d'Asie }\end{array}$ & $\begin{array}{l}\text { Amérique } \\
\text { latine } \\
\text { et Caraïbes }\end{array}$ & $\begin{array}{l}\text { Croissant } \\
\text { moyen- } \\
\text { oriental }\end{array}$ & $\begin{array}{l}\text { Anciennes } \\
\text { économies } \\
\text { socialistes } \\
\text { d'Europe }\end{array}$ & $\begin{array}{l}\text { Économies } \\
\text { de marché } \\
\text { bien } \\
\text { établies }\end{array}$ \\
\hline Population (millions d'habitants) & 5267 & 510 & 850 & 1134 & 683 & 444 & 503 & 346 & 798 \\
\hline $\begin{array}{l}\text { Maladies transmissibles } \\
\text { Tuberculose } \\
\text { MST et SIDA } \\
\text { Affections diarrhéiques } \\
\text { Maladies de l'enfance évitables par } \\
\text { vaccination } \\
\text { Paludisme } \\
\text { Affections dues à des vers parasites } \\
\text { Infections respiratoires } \\
\text { Affections liées à la maternité } \\
\text { Causes périnatales } \\
\text { Divers }\end{array}$ & $\begin{array}{r}45,8 \\
3,4 \\
3,8 \\
7,3 \\
5,0 \\
2,6 \\
1,8 \\
9,0 \\
2,2 \\
7,3 \\
3,5\end{array}$ & $\begin{array}{r}71,3 \\
4,7 \\
8,8 \\
10,4 \\
9,6 \\
10,8 \\
1,8 \\
10,8 \\
2,7 \\
7,1 \\
4,6\end{array}$ & $\begin{array}{r}50,5 \\
3,7 \\
2,7 \\
9,6 \\
6,7 \\
0,3 \\
0,9 \\
10,9 \\
2,7 \\
9,1 \\
4,0\end{array}$ & $\begin{array}{r}25,3 \\
2,9 \\
1,7 \\
2,1 \\
0,9 \\
* \\
3,4 \\
6,4 \\
1,2 \\
5,2 \\
1,4\end{array}$ & $\begin{array}{r}48,5 \\
5,1 \\
1,5 \\
8,3 \\
4,5 \\
1,4 \\
3,4 \\
11,1 \\
2,5 \\
7,4 \\
3,3\end{array}$ & $\begin{array}{r}42,2 \\
2,5 \\
6,6 \\
5,7 \\
1,6 \\
0,4 \\
2,5 \\
6,2 \\
1,7 \\
9,1 \\
5,8\end{array}$ & $\begin{array}{r}51,0 \\
2,8 \\
0,7 \\
10,7 \\
\\
6,0 \\
0,2 \\
0,4 \\
11,5 \\
2,9 \\
10,9 \\
4,9\end{array}$ & $\begin{array}{l}8,6 \\
0,6 \\
1,2 \\
0,4 \\
\\
0,1 \\
* \\
* \\
2,6 \\
0,8 \\
2,4 \\
0,6\end{array}$ & $\begin{array}{l}9,7 \\
0,2 \\
3,4 \\
0,3 \\
\\
0,1 \\
* \\
* \\
2,6 \\
0,6 \\
2,2 \\
0,5\end{array}$ \\
\hline $\begin{array}{l}\text { Maladies non transmissibles } \\
\text { Cancer } \\
\text { Carences nutritionnelles } \\
\text { Troubles neuro-psychiatriques } \\
\text { Affections cérébro-vasculaires } \\
\text { Cardiopathies ischémiques } \\
\text { Congestions pulmonaires } \\
\text { Divers }\end{array}$ & $\begin{array}{r}42,2 \\
5,8 \\
3,9 \\
6,8 \\
3,2 \\
3,1 \\
1,3 \\
18,9\end{array}$ & $\begin{array}{r}19,4 \\
1,5 \\
2,8 \\
3,3 \\
1,5 \\
0,4 \\
0,2 \\
9,7\end{array}$ & $\begin{array}{r}40,4 \\
4,1 \\
6,2 \\
6,1 \\
2,1 \\
2,8 \\
0,6 \\
18,5\end{array}$ & $\begin{array}{r}58,0 \\
9,2 \\
3,3 \\
8,0 \\
6,3 \\
2,1 \\
5,5 \\
23,6\end{array}$ & $\begin{array}{r}40,1 \\
4,4 \\
4,6 \\
7,0 \\
2,1 \\
3,5 \\
0,5 \\
17,9\end{array}$ & $\begin{array}{r}42,8 \\
5,2 \\
4,6 \\
8,0 \\
2,6 \\
2,7 \\
0,7 \\
19,1\end{array}$ & $\begin{array}{r}36,0 \\
3,4 \\
3,7 \\
5,6 \\
2,4 \\
1,8 \\
0,5 \\
18,7\end{array}$ & $\begin{array}{r}74,8 \\
14,8 \\
1,4 \\
11,1 \\
8,9 \\
13,7 \\
1,6 \\
23,4\end{array}$ & $\begin{array}{r}78,4 \\
19,1 \\
1,7 \\
15,0 \\
5,3 \\
10,0 \\
1,7 \\
25,6\end{array}$ \\
\hline $\begin{array}{l}\text { Affections traumatiques } \\
\text { Accidents de la circulation } \\
\text { Blessures volontaires } \\
\text { Divers }\end{array}$ & $\begin{array}{r}11,9 \\
2,3 \\
3,7 \\
5,9\end{array}$ & $\begin{array}{l}9,3 \\
1,3 \\
4,2 \\
3,9\end{array}$ & $\begin{array}{l}9,1 \\
1,1 \\
1,2 \\
6,8\end{array}$ & $\begin{array}{r}16,7 \\
2,3 \\
5,1 \\
9,3\end{array}$ & $\begin{array}{r}11,3 \\
2,3 \\
3,2 \\
5,8\end{array}$ & $\begin{array}{r}15,0 \\
5,7 \\
4,3 \\
5,0\end{array}$ & $\begin{array}{r}13,0 \\
3,3 \\
5,2 \\
4,6\end{array}$ & $\begin{array}{r}16,6 \\
3,7 \\
4,8 \\
8,1\end{array}$ & $\begin{array}{r}11,9 \\
3,5 \\
4,0 \\
4,3\end{array}$ \\
\hline Total & 100 & 100 & 100 & 100 & 100 & 100 & 100 & 100 & 100 \\
\hline $\begin{array}{l}\text { Millions d'AVCI } \\
\text { Nombre équivalent de décès } \\
\text { de nourrissons (millions) } \\
\text { Nombre d'AVCI pour } 1000 \text { habitants }\end{array}$ & $\begin{array}{l}1362 \\
42,0 \\
259\end{array}$ & $\begin{array}{r}293 \\
9,0 \\
575\end{array}$ & $\begin{array}{r}292 \\
9,0 \\
344\end{array}$ & $\begin{array}{r}201 \\
6,2 \\
178\end{array}$ & $\begin{array}{r}177 \\
5,5 \\
260\end{array}$ & $\begin{array}{r}103 \\
3,2 \\
233\end{array}$ & $\begin{array}{r}144 \\
4,4 \\
286\end{array}$ & $\begin{array}{r}58 \\
1,8 \\
168\end{array}$ & $\begin{array}{r}94 \\
2,9 \\
117\end{array}$ \\
\hline
\end{tabular}

* Moins de 0,05\%. AVCl = années de vie corrigées du facteur invalidité; MST = maladies sexuellement transmissibles.

Source: Données de la Banque Mondiale. Rapport sur le développement dans le monde 1993.

tion. Ou encore, pour prendre un exemple extrême, si la population entière de la planète devait s'éteindre en un an, la charge de morbidité pour 1000 habitants serait supérieure à 20000 AVCI pour cette année-là. Il n'y a donc pas d'échelle absolue par rapport à laquelle mesurer la charge de morbidité globale pour 1000 habitants, les seules comparaisons qui aient un sens sont celles que l'on peut faire entre catégories de régions, facteurs de risque, groupes de maladies ou sexes. Le Tableau I indique comment la charge de morbidité globale se décompose par catégorie d'affections et par région démographique. La méthode retenue pour calculer la charge de morbidité globale peut aussi être employée pour suivre les progrès de la santé publique dans $\mathrm{m} / \mathrm{s} n^{\circ} 11$ vol. 9, novembre 93

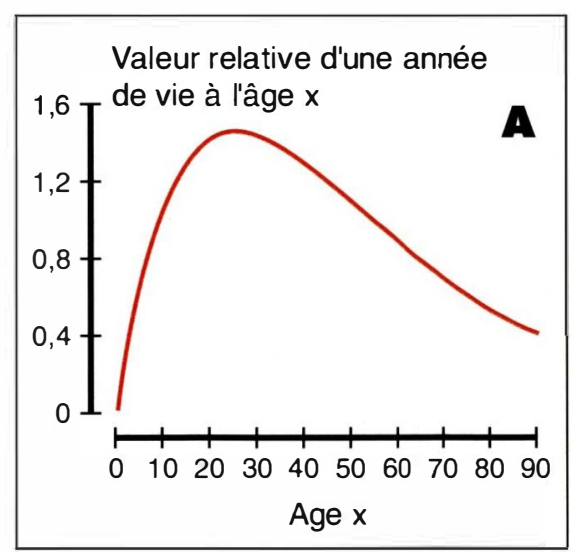

tel ou tel pays d'après l'évolution de la charge de morbidité dans ce pays. Des plans ont été esquissés pour la réalisation d'études selon cette méthode en Afrique du Sud, au Costa Rica et en Inde (dans l'État de l'Andhra Pradesh)

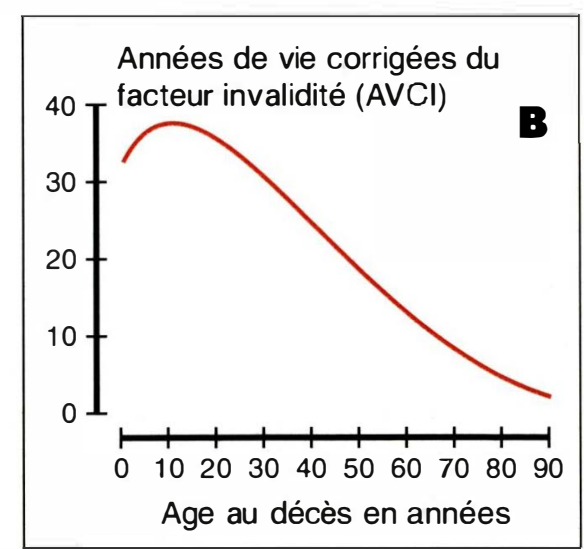

Figure 1. Évolution par âge des pondérations en fonction de l'áge des pertes d'années de vie corrigées du facteur invalidité (AVCI).

A : valeur relative d'une année de vie à l'âge x. B: AVCl perdues pour cause de décès à différents âges chez les femmes. 